\title{
Instrumental tactile diagnostics in robot-assisted surgery
}

This article was published in the following Dove Press journal:

Medical Devices: Evidence and Research

31 October 2016

Number of times this article has been viewed

\author{
Rozalia F Solodova ${ }^{1,2}$ \\ Vladimir V Galatenkol,2 \\ Eldar R Nakashidze ${ }^{3}$ \\ Igor L Andreytsev ${ }^{3}$ \\ Alexey V Galatenko' \\ Dmitriy K Senchik ${ }^{2}$ \\ Vladimir M Staroverov' \\ Vladimir E Podolskii ${ }^{1,2}$ \\ Mikhail E Sokolov ${ }^{1,2}$ \\ Victor A Sadovnichy ${ }^{1,2}$ \\ 'Faculty of Mechanics and \\ Mathematics, ${ }^{2}$ Institute of \\ Mathematical Studies of Complex \\ Systems, Lomonosov Moscow State \\ University, ${ }^{3}$ I st Surgery Department, \\ Clinical Hospital 3I, Moscow, Russia
}

Correspondence: Rozalia F Solodova I-46 Leninskiye Gory, Office I45, GSP-I, Moscow I I 999I, Russia

$\mathrm{Tel}+79199666152$

Fax +7 4959394536

Email rozaliya@solodov.org
Background: Robotic surgery has gained wide acceptance due to minimizing trauma in patients. However, the lack of tactile feedback is an essential limiting factor for the further expansion. In robotic surgery, feedback related to touch is currently kinesthetic, and it is mainly aimed at the minimization of force applied to tissues and organs. Design and implementation of diagnostic tactile feedback is still an open problem. We hypothesized that a sufficient tactile feedback in robot-assisted surgery can be provided by utilization of Medical Tactile Endosurgical Complex (MTEC), which is a novel specialized tool that is already commercially available in the Russian Federation. MTEC allows registration of tactile images by a mechanoreceptor, real-time visualization of these images, and reproduction of images via a tactile display.

Materials and methods: Nine elective surgeries were performed with da Vinci ${ }^{\mathrm{TM}}$ robotic system. An assistant performed tactile examination through an additional port under the guidance of a surgeon during revision of tissues. The operating surgeon sensed registered tactile data using a tactile display, and the assistant inspected the visualization of tactile data. First, surgeries where lesion boundaries were visually detectable were performed. The goal was to promote cooperation between the surgeon and the assistant and to train them in perception of the tactile feedback. Then, instrumental tactile diagnostics was utilized in case of visually undetectable boundaries.

Results: In robot-assisted surgeries where lesion boundaries were not visually detectable, instrumental tactile diagnostics performed using MTEC provided valid identification and localization of lesions. The results of instrumental tactile diagnostics were concordant with the results of intraoperative ultrasound examination. However, in certain cases, for example, thoracoscopy, ultrasound examination is inapplicable, while MTEC-based tactile diagnostics can be efficiently utilized.

Conclusion: The study proved that MTEC can be efficiently used in robot-assisted surgery allowing correct localization of visually undetectable lesions and visually undetectable boundaries of pathological changes of tissues.

Keywords: tactile feedback, instrumental palpation, Medical Tactile Endosurgical Complex, tactile lesion localization

\section{Introduction}

Palpation helps to identify and localize pathological changes of tissues during open surgeries. The introduction of minimally invasive endoscopic surgery almost 30 years ago led to substitution of a tactile feedback by a kinesthetic feedback. A kinesthetic feedback is transmitted through instruments and related to force and positions of muscles and joints, while a tactile (or cutaneous) feedback is sensed by hands (in particular, fingers) and related to skin. 
Widely developing robot-assisted surgery currently lacks feedback related to touch. Increased freedom of manipulation, access to tight spaces, and stereoscopic displays are the benefits which promote the continuous growth of the segment of robot-assisted surgery. ${ }^{1-3}$ However, the lack of tactile feedback is an essential limiting factor for the expansion of this segment. ${ }^{46}$ Certain studies were performed aimed at the improvement of force feedback in minimally invasive or robotic surgeries. ${ }^{7,8}$ However, the problem of tactile feedback was not adequately studied: the results were either preliminary from the viewpoint of clinical applications ${ }^{9-12}$ or not focused on surgery. ${ }^{13,14}$ At the same time, tactile feedback is essential for control of tissue interaction ${ }^{15}$ and cannot be completely substituted by visual or audio feedback. ${ }^{16,17}$

In robot-assisted surgery, feedback related to touch is currently kinesthetic, and assessment in most research is based on force applied to tissues and organs. ${ }^{18}$ Recent studies in this field showed that kinesthetic feedback provides a decrease of grasping force, which leads to lower tissue damage. ${ }^{19}$ However, design and implementation of diagnostic tactile feedback is still an open problem.

We hypothesized that a sufficient tactile feedback in robot-assisted surgery can be provided by utilization of Medical Tactile Endosurgical Complex (MTEC), which is a novel specialized tool that is already commercially available in the Russian Federation. ${ }^{20}$ MTEC allows registration of tactile images by a mechanoreceptor, real-time visualization of these images, and reproduction of images via a tactile display.

Previously, MTEC was successfully utilized in traditional endoscopic surgeries, ${ }^{21}$ but its applicability in a framework of robotic surgery remained unstudied. Our study showed that after a short training, one can correctly localize visually undetectable pathological changes using MTEC.

\section{Materials and methods MTEC}

MTEC consists of a tactile mechanoreceptor (Figure 1A), a tactile display (Figure 1C), and a computer. An operating head of a tactile mechanoreceptor (Figure 1B) contains seven pressure sensors which perform registration and wirelessly transmit data 100 times per second. A computer receives registered values, performs scaling, online visualization, and additional processing, and transmits data to a tactile display. Tactile display dynamically reproduces tactile data in real time; the data can be read simply by a finger.

Sensors of a tactile mechanoreceptor provide pressure measurements with resolution of an order of 10 Pa per least significant digit, and error/noise magnitude of an order of

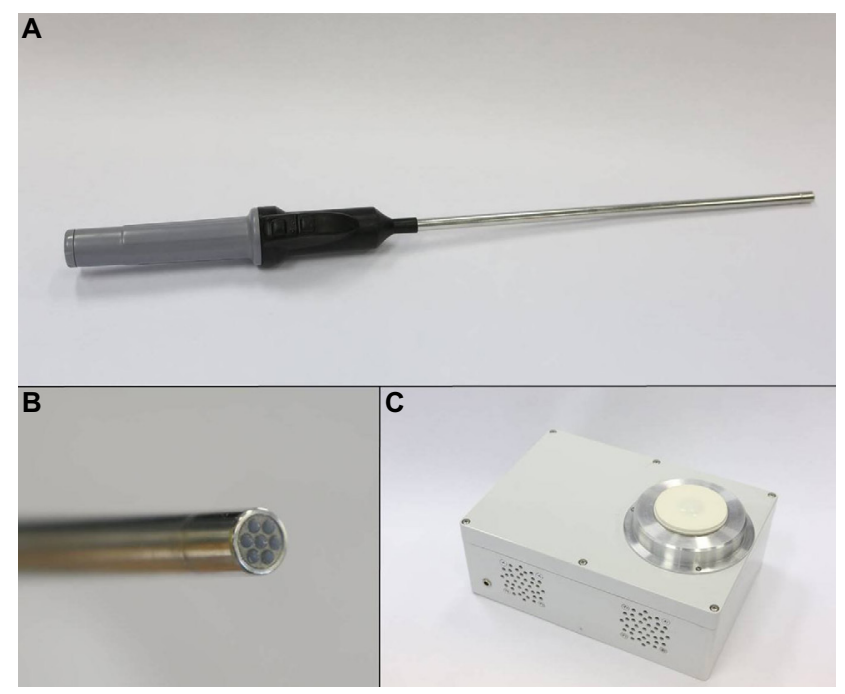

Figure I Medical tactile endosurgical complex. (A) A tactile mechanoreceptor (general view). (B) An operating head of a tactile mechanoreceptor. (C) A tactile display.

$100 \mathrm{~Pa}$. A membrane that separates sensors and the examined surface leads to a reduction of measurement resolution by one order of magnitude. However, this reduction and this error/ noise magnitude are acceptable, as tactile reproduction and visualization utilize a discretization with just 256 levels. This number of levels is completely sufficient for perception. The lowest discretization level corresponds to zero pressure (ie, no contact with an examined surface), and the highest level corresponds to pressures equal to or exceeding $P_{\max }$. The value of $P_{\max }$ can be adjusted according to the stiffness of an examined tissue, but its typical values have an order of $30 \mathrm{kPa}$.

Visualization of a tactile frame is based on a linear conversion of a discretized pressure level into a color code in a standard green-blue-red color scale where green corresponds to zero pressure, blue corresponds to medium pressure values, and red corresponds to high pressure values in comparison with $P_{\max }$ (Figure 2G). Processing of registered tactile data includes analysis aimed at automated detection of heterogeneities. ${ }^{22}$

MTEC-based tactile examination of an area is performed by sequential rectilinear presses aimed at detection of a lesion boundary. After a boundary segment is detected, further examination is focused on the complete localization of the boundary and thus localization of a lesion. As the diameter of an operating head of a mechanoreceptor is $1 \mathrm{~cm}$, the precision of the localization has an order of $5 \mathrm{~mm}$. Pressing force is applied and controlled manually by an operator (in case of robot-assisted surgery, by an assistant).

Boundary detection and localization are performed based on the tactile reproduction and visualization of registered tactile images. A press on a homogeneous area results in 


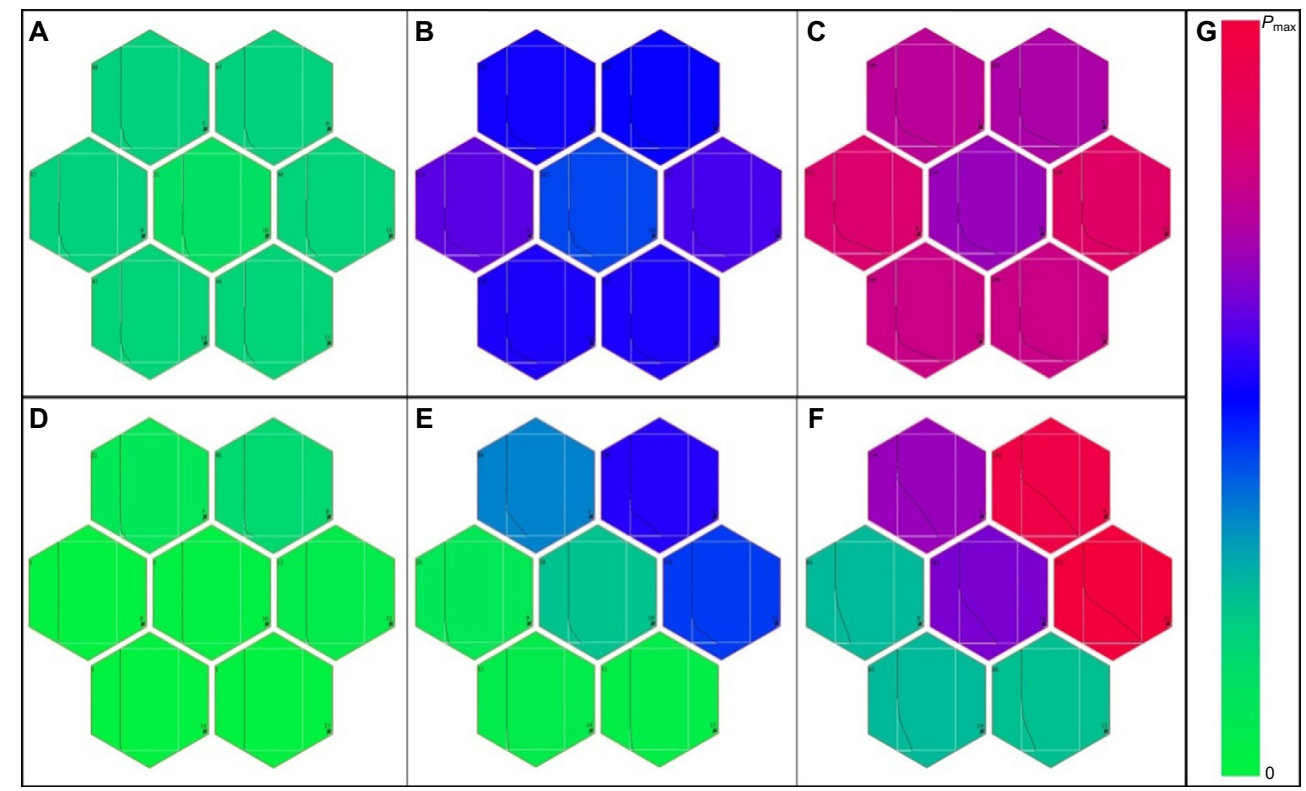

Figure 2 Visualization of tactile frames. (A-C) Tactile frames registered during one press on a homogeneous sample; pressing force grows from (A) to (C). (D-F) Tactile frames registered during one press on a boundary; pressing force grows from (D) to (F). (G) A color scale used for color coding of discretized pressure levels. Each box of a frame is associated with one pressure sensor and contains color-coded value of current pressure and a curve representing previous measurements (measurement remoteness is plotted along the vertical axis, and horizontal axis gives a corresponding discretized pressure level).

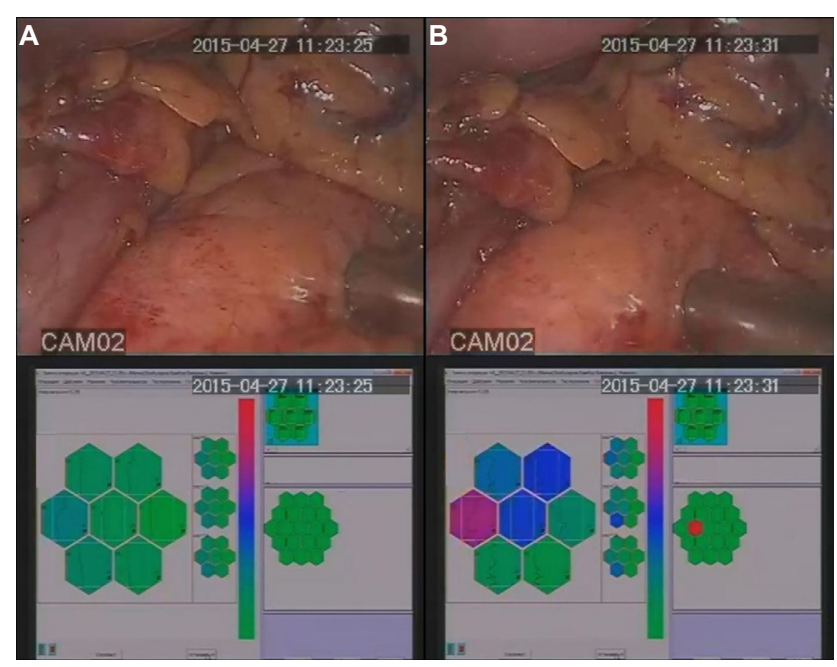

Figure 3 Tactile identification and localization of a visually undetectable lesion during a pancreas resection: simultaneously registered intraoperative video frames and tactile frames. (A) A press on a homogeneous area. (B) A press on the lesion boundary.

homogeneous tactile frames (Figures $2 \mathrm{~A}-\mathrm{C}$ and $3 \mathrm{~A}$ ), while a press on a boundary results in highly contrasting tactile frames (Figures 2D-F and 3B).

\section{MTEC utilization in robot-assisted surgery}

During the surgeries, an assistant performed tactile examination through an additional port under the guidance of a surgeon. The examination was performed at the tissue revision stage. The operating surgeon sensed registered tactile data using a tactile display (Figure 4A), and the assistant inspected the visualization of tactile data (Figure 4B). All
$\underline{A}$ B

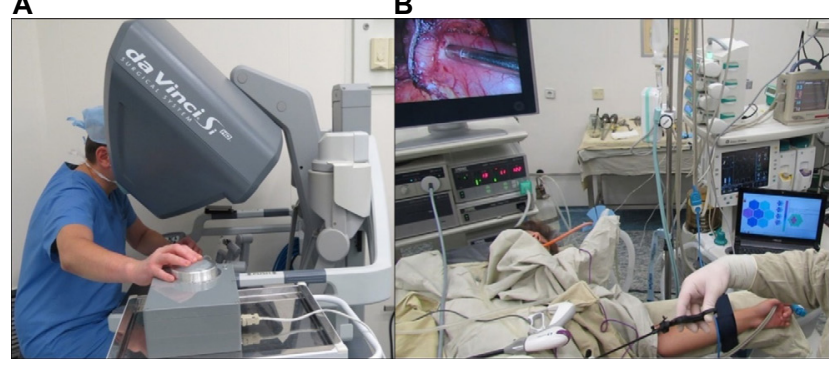

Figure 4 MTEC utilization during a robot-assisted surgery. (A) A surgeon sensing registered tactile data using a tactile display. (B) Intraoperative real-time visualization of registered tactile data.

surgeries were comprehensively registered. In particular, video, audio, and tactile data were recorded.

\section{Patients and surgeries}

The applicability of MTEC in robot-assisted surgery was tested from January 2015 to December 2015 in Moscow Clinical Hospital 31 (Russian Federation). The study was approved by the Ethics Committee of Clinical Hospital 31.

Nine elective surgeries were performed with da Vinci ${ }^{\mathrm{TM}}$ robotic system (Intuitive Surgical, Sunnyvale, CA, USA): two gastrectomies, two stomach resections, two resections of pancreas, two prostatectomies, and one right hemicolectomy. Patients' ages were from 30 to 76 years with an average of 55 years. Patients (six males and three females) signed an informed consent form before surgery which included having their depersonalized data used for medical scientific study/ 
research and described/presented in any medical scientific/ research paper.

First, surgeries where lesion boundaries were visually detectable were performed. Then, instrumental tactile diagnostics was used in case of visually undetectable boundaries.

In case of resection of pancreas, hemicolectomy and one stomach resection, tactile examination performed using MTEC was followed by an intraoperative ultrasound examination performed with Flex Focus Ultrasound Machine (BK Medical, Herlev, Denmark).

\section{Results}

MTEC was first tested on visually identifiable lesions of stomach and intestine (five cases). The goal of this stage was to confirm the protocol of tactile examination, to develop cooperation between the surgeon and the assistant, and to train them in perception of the tactile feedback. Then, instrumental tactile diagnostics was used in case of visually undetectable boundaries of pathological changes.

\section{Cases with visually detectable lesion boundaries (training)}

Five surgeries were performed at the training stage, namely two gastrectomies, two stomach resections, and a right hemicolectomy. The cases included three stomach adenocarcinomas, one ascending colon adenocarcinoma, and one gastrointestinal stromal tumor (GIST).

Instrumental tactile examination was performed starting from the proximal end of a lesion toward the boundary. After the boundary was reached, instrumental palpation was performed clockwise until the complete localization of the lesion was achieved. The surgeon received training in interpretation of tactile data read via a tactile display. In particular, he trained in boundaries identification based on these data. The assistant trained in perception of visualization of tactile data and force applied to the instrument during examination.

In case of GIST boundaries, identification based on instrumental tactile diagnostics was less apparent in comparison with the other cases. This result was concordant with the results of direct palpation by fingers of a surgically removed material.

\section{Cases with visually undetectable lesion boundaries}

After the training stage was completed, the instrumental tactile diagnostics was applied in cases of visually undetectable lesion boundaries. Four surgeries were performed: two prostatectomies and two resections of pancreas.
During tactile examination of the prostate, the whole gland was palpated along with adjacent tissues. In one case, more solid regions were detected in the left lobe and in the right lobe, which corresponded to adenocarcinoma of the left and right lobes of the prostate gland. In the other case, the lesion boundary could not be localized explicitly, but a more solid region was identified in the right lobe. The histological examination revealed micro-acinar carcinoma of the right lobe of the prostate.

During pancreas resection, instrumental tactile diagnostics was followed by an intraoperative ultrasound examination. In the first case (Figure 3), the instrumental tactile diagnostics revealed that pancreas was indurated along the entire length, with the most pronounced induration in the projection of the pancreas body, where a more solid region with a diameter of approximately $2 \mathrm{~cm}$ was detected and localized. Instrumental palpation of parapancreatic fiber revealed no pathological changes. The intraoperative ultrasound examination identified a lesion of size up to $27 \mathrm{~mm}$ with clear irregular boundaries in the body of the pancreas. The echotexture in the lesion region was evidently different from the echotexture of parenchyma of pancreas head and tail: it was heterogeneous, with a significant interlobular fibrosis. The histological examination revealed acinar cell carcinoma of the pancreas.

In the second case, the pancreas had a uniformly increased thickness along the entire length. The lesion boundaries could not be detected by instrumental tactile examination. The echotexture of the parenchyma was heterogeneous with multiple mixed hyperechoic and hypoechoic foci. The head and the body of the pancreas were incrementally removed. An urgent histological examination revealed pathological changes in the resected border, and hence, taking into consideration the results of intraoperative tactile and ultrasound diagnostics, duodenopancreatectomy was performed. The histological examination revealed intraductal papillary mucinous neoplasm with high-grade dysplasia.

Thus, in robot-assisted surgeries where lesion boundaries were not visually detectable, instrumental tactile diagnostics performed using MTEC allowed valid identification and localization of lesions. The results of instrumental tactile diagnostics were concordant with the results of intraoperative ultrasound examination.

\section{Discussion}

Tactile properties of abnormal tissues are essentially different from tactile properties of adjacent healthy tissues. In particular, elastic modulus of tumors is an order of magnitude higher than elastic modulus of healthy tissues. ${ }^{23,24}$ 
Thus, tactile examination is a natural and efficient method for identification and localization of pathological changes.

Instrumental registration and automated analysis of tactile images allow better diagnostics even if standard manual palpation is possible. For example, tactile examination of patients with a complaint of a breast mass resulted in sensitivity and specificity of identifying a dominant mass both equal to $88 \%$ when instrumental tactile imaging technology was utilized, while for standard physical palpations, both values were $81 \% .^{25}$

In endoscopic surgery, including robot-assisted surgery, instrumental tactile palpation is not a potential alternative or a supplement to a manual palpation, but it is the only way to perform tactile examination of tissues. To the best of our knowledge, despite a number of reports on perspective technologies, ${ }^{9-12,19,26}$ MTEC is currently the only commercially available medical device for intraoperative registration of tactile images. A previous study showed that for a simple testing stand, MTEC allowed correct localization of $95 \%$ of small heterogeneities $(95 \%$ confidence interval for the correct detection rate: $[0.83,0.99]){ }^{22}$ The experiment used a mechanoreceptor with 19 pressure sensors and an operating head with a diameter of $2 \mathrm{~cm}$. The result was concordant with the result of MTEC utilization in detection of pulmonary lesions during thoracoscopy where detection rate was 0.81 (95\% confidence interval: $[0.62,0.94]) .{ }^{21}$ At the same time, for conventional instrumental palpation by lung forceps, detection rate was only 0.37 . Thus, for $71 \%$ of lesions impalpable by lung forceps, MTEC allowed correct lesion detection and prevented conversion during surgery. The difference between efficiency of tactile examination by MTEC and by conventional lung forceps is statistically significant with a $P$-value of 0.0015 (McNemar's test). The latter study was performed using mechanoreceptors with seven pressure sensors and an operating head with a diameter of $1 \mathrm{~cm}$, that is, the type that was utilized in the described robot-assisted surgeries.

Our present study proves that MTEC tactile examination is also efficient in a framework of robot-assisted surgery. It provided correct results in all the cases, including four cases of visually undetectable lesion boundaries. The results of MTEC tactile examination were concordant with the results of intraoperative ultrasound examination, which is known to be a highly efficient method for detection of small lesions in abdomen. However, in certain cases, for example, thoracoscopy, ultrasound examination is inapplicable, while MTEC-based tactile diagnostics can be efficiently utilized.

The further development of MTEC which can essentially improve its usability is a design of a flexible tip with a larger area in comparison with the current operating head and hence with a larger number of pressure sensors. In particular, this design would speed up tactile examination and simplify the examination of tissues with a limited rectilinear access. The current version of a tactile mechanoreceptor is hardly applicable for such tissues. This design would also increase the feasibility of nontrivial automated analysis of tactile images including pattern recognition. Seven sensors allow a reliable identification and localization of boundaries but are not sufficient for a comprehensive automated analysis of registered tactile images.

\section{Conclusion}

Our study proved that MTEC can be efficiently used in robotassisted surgery allowing correct localization of visually undetectable lesions and visually undetectable boundaries of pathological changes of tissues.

\section{Acknowledgments}

The research was supported by the Russian Science Foundation (project 16-11-00058 "The development of methods and algorithms for automated analysis of medical tactile information and classification of tactile images"). The authors thank Dr Vladimir M Budanov for valuable comments on technical specification of MTEC.

\section{Disclosure}

The authors report no conflicts of interest in this work.

\section{References}

1. Ng AT, Tam PC. Current status of robot-assisted surgery. Hong Kong Med J. 2014;20(3):241-250.

2. Khosla A, Wagner AA. Robotic surgery of the kidney, bladder, and prostate. Surg Clin North Am. 2016;96(3):615-636.

3. Bouquet de Joliniere J, Librino A, Dubuisson JB, et al. Robotic surgery in gynecology. Front Surg. 2016;3:26.

4. Okamura AM. Haptic feedback in robot-assisted minimally invasive surgery. Curr Opin Urol. 2009;19(1):102-107.

5. Enayati N, De Momi E, Ferrigno G. Haptics in robot-assisted surgery: challenges and benefits. IEEE Rev Biomed Eng. 2016;9:49-65.

6. Leal Ghezzi T, Campos Corleta O. 30 years of robotic surgery. World $J$ Surg. 2016;40(10):2550-2557.

7. Hamed A, Chun Tang S, Ren H, et al. Advances in haptics, tactile sensing, and manipulation for robot-assisted minimally invasive surgery, noninvasive surgery, and diagnosis. J Robot. 2012;2012:412816.

8. Ehrampoosh S, Dave M, Kia MA, Rablau C, Zadeh MH. Providing haptic feedback in robot-assisted minimally invasive surgery: a direct optical force-sensing solution for haptic rendering of deformable bodies. Comput Aided Surg. 2013;18(5-6):129-141.

9. Culmer P, Barrie J, Hewson R, et al. Reviewing the technological challenges associated with the development of a laparoscopic palpation device. Int J Med Robot. 2012;8(2):146-159.

10. Ottermo MV, Stavdahl Ø, Johansen TA. A remote palpation instrument for laparoscopic surgery: design and performance. Minim Invasive Ther Allied Technol. 2009;18(5):259-272. 
11. Afshari E, Najarian S, Simforoosh N, Hajizade Farkoush S. Design and fabrication of a novel tactile sensory system applicable in artificial palpation. Minim Invasive Ther Allied Technol. 2011;20(1):22-29.

12. Abouei Mehrizi A, Moini M, Afshari E, Kadkhodapour J, Sadjadian A, Najarian S. Application of artificial palpation in vascular surgeries for detection of peripheral arterial stenosis. J Med Eng Technol. 2014;38(4):169-178.

13. Egorov V, Sarvazyan AP. Mechanical imaging of the breast. IEEE Trans Med Imaging. 2008;27(9):1275-1287.

14. Egorov V, van Raalte $\mathrm{H}$, Sarvazyan AP. Vaginal tactile imaging. IEEE Trans Biomed Eng. 2010;57(7):1736-1744.

15. Lim SC, Lee HK, Park J. Role of combined tactile and kinesthetic feedback in minimally invasive surgery. Int J Med Robot. 2015;11(3): 360-374.

16. Meccariello G, Faedi F, AlGhamdi S, et al. An experimental study about haptic feedback in robotic surgery: may visual feedback substitute tactile feedback? J Robot Surg. 2016;10(1):57-61.

17. Koehn JK, Kuchenbecker KJ. Surgeons and non-surgeons prefer haptic feedback of instrument vibrations during robotic surgery. Surg Endosc. 2015;29(10):2970-2983.

18. Culjat MO, King CH, Franco ML, et al. A tactile feedback system for robotic surgery. Conf Proc IEEE Eng Med Biol Soc. 2008;2008: 1930-1934.
19. Wottawa CR, Genovese B, Nowroozi BN, et al. Evaluating tactile feedback in robotic surgery for potential clinical application using an animal model. Surg Endosc. 2016;30(8):3198-3209.

20. Sadovnichy V, Gabidullina R, Sokolov M, Galatenko V, Budanov V, Nakashidze E. Haptic device in endoscopy. Stud Health Technol Inform. 2014;196:365-368.

21. Barmin V, Sadovnichy V, Sokolov M, Pikin O, Amiraliev A. An original device for intraoperative detection of small indeterminate nodules. Eur J Cardiothorac Surg. 2014;46(6):1027-1031.

22. Solodova R, Staroverov V, Galatenko V, et al. Automated detection of heterogeneity in medical tactile images. Stud Health Technol Inform. 2016;220:383-389.

23. Yallapu MM, Katti KS, Katti DR, et al. The roles of cellular nanomechanics in cancer. Med Res Rev. 2015;35(1):198-223.

24. Fenner J, Stacer AC, Winterroth F, Johnson TD, Luker KE, Luker GD. Macroscopic stiffness of breast tumors predicts metastasis. Sci Rep. 2014;4:5512.

25. Kaufman CS, Jacobson L, Bachman BA, Kaufman LB. Digital documentation of the physical examination: moving the clinical breast exam to the electronic medical record. Am J Surg. 2006;192(4):444-449.

26. Schostek S, Zimmermann M, Schurr MO, Prosst RL. Design and performance of a low-cost telemetric laparoscopic tactile grasper. Surg Innov. 2016;23(3):291-297.
Medical Devices: Evidence and Research

\section{Publish your work in this journal}

Medical Devices: Evidence and Research is an international, peerreviewed, open access journal that focuses on the evidence, technology, research, and expert opinion supporting the use and application of medical devices in the diagnosis, monitoring, treatment and management of clinical conditions and physiological processes. The identification of novel
Dovepress

devices and optimal use of existing devices which will lead to improved clinical outcomes and more effective patient management and safety is a key feature. The manuscript management system is completely online and includes a quick and fair peer-review system. Visit http://www. dovepress.com/testimonials.php to read real quotes from authors. 